\title{
Multielectrode intrafascicular and extraneural stimulation
}

\author{
$\begin{array}{llll}\text { P. H. Veltink } & \text { J. A. van Alsté } & \text { H. B. K. Boom }\end{array}$ \\ Biomedical Engineering Division, Department of Electrical Engineering, University of Twente \\ PO Box 217, 7500 AE Enschede, The Netherlands
}

\begin{abstract}
The relationship between nerve stimulation, pulse amplitude and isometric muscle force was measured to investigate recruitment of motor units. Force addition experiments were performed to obtain insight in the intersection of motor unit groups recruited by different electrodes. Intrafascicular and extraneural multielectrode configurations were used for nerve stimulation. Experiments were performed on rats. The common peroneal nerve was stimulated and the forces of the tibial anterior and extensor digitorum longus muscles were measured isometrically. Recruitment was more stable for intrafascicular electrodes than for extraneural electrodes. Especially for intrafascicular electrodes no strict inverse recruitment was observed. Force addition experiments indicated that small overlap of recruited motor unit groups occurred more often for intrafascicular than for extraneural electrodes.
\end{abstract}

Keywords-Extraneural electrodes, Intrafascicular electrodes, Multielectrode nerve stimulation, Nerve fibre recruitment

Med. \& Biol. Eng. \& Comput., 1989, 27, 19-24

\section{Introduction}

Contractions of skeletal muscles can be induced by artificial electrical stimulation of peripheral nerves, a technique which may be of great clinical use. However, an adequate grading of muscle force, low muscle fatigue and sufficient muscle selectivity are not self-evident in artificial nerve stimulation. Fluctuations in nerve fibre excitation threshold (GoRMAN and MorTimer, 1983; VerVEen and DERKSEN, 1968) and a steep relationship between stimulation pulse amplitude and muscle force result in limited force grading possibilities. Inverse recruitment order of motor units (big motor units fire first) also diminish muscle grading and increase muscle fatigue (PETROFSKY, 1978; PETROFSKY and PhILliPS, 1981; FANG and MorTIMER, 1987). Depending on the position along the nerve, motoneurons innervating different muscles may mix their axons in the same nerve and even in the same fascicle. This hampers selective stimulation of muscles.

In recent years advances have been made to alleviate some of the above problems. GORMAN and MORTIMER (1983) investigated the influence of stimulation pulse parameters on muscle grading and recruitment order. The application of nerve fibre blocking to restore natural recruitment order and thus decrease muscle fatigue was investigated by Petrofsky (1978), Petrofsky and PhILLIPS (1981), SOLOMONOW et al. (1983) and FANG and MORTIMER (1987). Sequential stimulation of motor unit groups using multielectrode configurations also was found to reduce muscle fatigue (Holle et al., 1974; Petrofsky, 1979). Furthermore, multielectrode configurations can be used for stimulating different muscles from the same nerve position (Thoma et al., 1978; MCNeal and Bowman, 1985; TALONEN et al., 1985). Configurations with multi-

First received 15th March and in final form 31st August 1988

(C) IFMBE: 1989 electrodes are only useful when overlap of these groups is limited.

In the present study the influence of electrode position (extraneural or intrafascicular) on muscle force grading, recruitment characteristics and overlap of recruited motor unit groups was investigated. Extraneural and intrafascicular electrode configurations were compared in rats.

\section{Methods}

\subsection{Experimental methods}

Rats (Wistar, male, $3-4$ months old, $0.30-0.35 \mathrm{~kg}$ ) were anaesthetised by intraperitoneal injection of pentobarbital sodium. The initial dose was $70 \mathrm{mg} \mathrm{kg}^{-1}$. On the average an additional $15 \mathrm{mg} \mathrm{kg}^{-1}$ was given every half-hour. Atropine was given subcutaneously.

In the right hind limb the common peroneal nerve was stimulated just below the bifurcation of the sciatic nerve into the tibial nerve and the common peroneal nerve (Fig. 1). The nerve was stimulated monopolarly with one electrode at a time, using cathodic monophasic rectangular current pulses with a width of $60 \mu \mathrm{s}$. Pulse amplitude was varied. A passive electrode was placed in between muscles outside the stimulation region. Two electrode configurations were examined for stimulation. First, an intrafascicular configuration consisting of four $25 \mu \mathrm{m}$ stainless-steel wires was isolated up to the tip and placed $100 \mu \mathrm{m}$ apart (Fig. 1a). Single intrafascicular wire electrodes $(25 \mu \mathrm{m}$ stainless-steel) were used for recruitment curve measurements. Insertion of the intrafascicular electrodes was done by making an incision in the epineurium and piercing the perineurium. The damage to the perineurium was minimised to prevent the intrafascicular substance from bulging out. However, when this would occur the electrodes were inserted in the nerve again at a position closer 


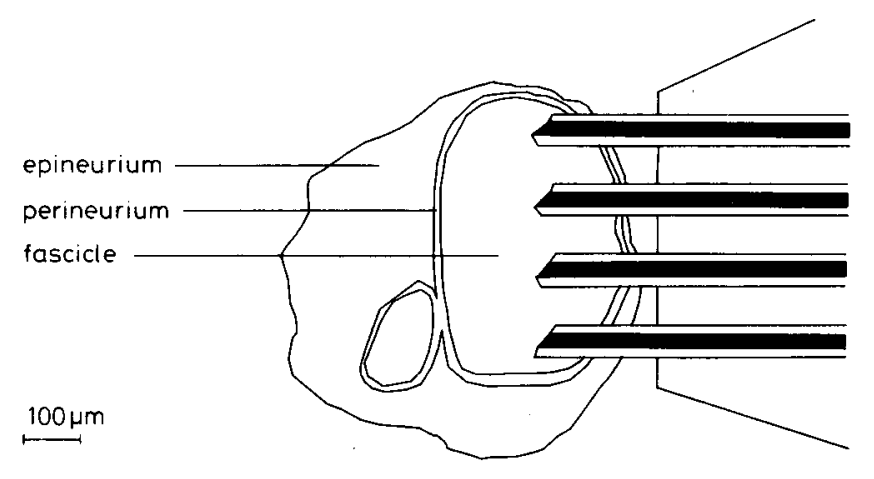

$a$

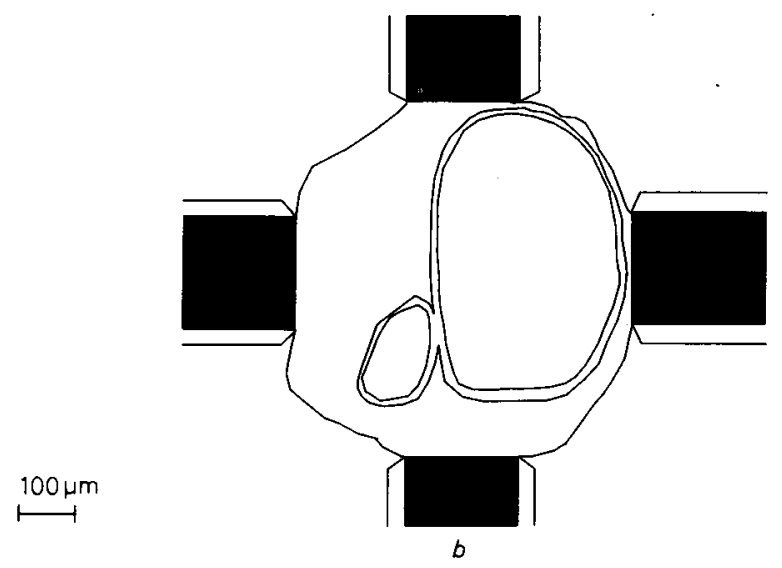

Fig. 1 The electrode configurations and the stimulated nerve (drawn to scale). (a) Intrafascicular electrode configuration, consisting of four $25 \mu \mathrm{m}$ diameter stainless-steel wires isolated upto the tip and placed $100 \mu \mathrm{m}$ apart; $(b)$ extraneural electrode configuration consisting of four $200 \mu \mathrm{m}$ diameter stainless-steel wires. The nerve is drawn from a photograph of the cross-section of the common peroneal nerve of the rat. Diameters of the nerve fibres vary between $5 \mu \mathrm{m}$ and $20 \mu \mathrm{m}$

to the muscle. The second electrode configuration was an extraneural configuration consisting of four $200 \mu \mathrm{m}$ diameter stainless-steel wires (Fig. $1 b$ ). The common peroneal nerve of the rat is a rather small nerve compared with human nerves and contains one main fascicle, sometimes with an additional smaller one. The four electrodes of the extraneural or intrafascicular electrode configuration were placed in one normal cross-section of the nerve. The nerve at this position was electrically isolated by lifting it up from the surrounding tissue over a distance of about 10 $20 \mathrm{~mm}$. The nerve was surrounded with paraffin to prevent it from drying. The stimulation region was chosen such that the nerve was not mechanically influenced by contraction of the stimulated muscles.

The forces of the tibialis anterior muscle (TA) and the extensor digitorum longus muscle (EDL) were measured isometrically. The femur was fixated and the insertio tendons were cut and fixed to isometric force transducers. The muscles were prepared free from the surrounding tissue except for their blood and nerve supply. In this way, mechanical interaction between muscles and surrounding tissue was minimised. Muscle temperature $\left(36-38^{\circ} \mathrm{C}\right)$ was controlled by a warm air stream across the muscle. The air was almost saturated with water vapour (WALLINGA-DE JONGE et al., 1980). Both muscles are innervated by the common peroneal nerve (GREENE, 1968). The nerve was not cut proximal to the place of stimulation in order to maintain its nutrition. No spinal reflexes were observed during the stimulation experiments.

\subsection{Recruitment curve measurements}

In three rats the relationship between stimulation pulse amplitude and isometric muscle force was measured. The nerve was stimulated at a frequency of 0.3 or $0.7 \mathrm{~Hz}$, which resulted in force twitches. The twitch amplitudes of TA and EDL were measured simultaneously. Stimulus pulses were stepwise varied in amplitude from low to distinctly high values. At each pulse amplitude, stimulation was repeated 5 or 7 times.

The relation between twitch amplitudes and pulse amplitude is represented by the recruitment curve. Above a threshold pulse amplitude twitch force amplitudes change from zero to a maximum. The 'narrowness' $S$ of a recruitment curve was defined as the quotient of the threshold stimulation pulse amplitude $I_{t}$ at which 5 per cent of the maximal twitch force was reached, and the pulse amplitude $I_{h}$ at which half-maximum twitch force was reached:

$$
S=\frac{I_{t}}{I_{h}}
$$

In this evaluation the average twitch amplitude measured for each stimulation pulse amplitude was considered, and linear interpolation between these values was performed. Only recruitment curves measured up to or further than half maximum twitch force were evaluated.

\subsection{Force addition measurement}

Force addition of the TA was measured in four rats and considered as an indication of overlap of recruited motor unit groups. The common peroneal nerve was stimulated submaximally during about $0.5 \mathrm{~s}$ at $70 \mathrm{~Hz}$ at one or at two electrodes, resulting in a near-tetanic contraction. After the application of a stimulus train at each electrode separately, stimulus trains were applied to both electrodes simultaneously. The individual stimulus pulses of the trains on the two electrodes were alternated to minimise electrical interference in the nerve and to obtain a comparable initial state of the nerve fibres before each stimulation pulse. Near-tetanic contractions were evaluated, because in isometric force measurements tetanic force levels are less influenced by the compliance of series elastic components in the muscle. In this situation series compliance is low, which minimises the influence of the velocity dependence of the muscle contractile components (WILKIE, 1950).

Each muscle force registration was averaged between $70 \mathrm{~ms}$ and $370 \mathrm{~ms}$ after the onset of stimulation. If $F_{s}$ denotes the mean force during simultaneous stimulation, and $F_{l}$ and $F_{h}$ the mean forces during stimulation on each electrode separately, of which $F_{h}$ is the higher force, the results were accepted for further evaluation under the following conditions:

(a) $F_{l}>0.3 F_{h}$

(b) $F_{l}$ as well as $F_{h}$ did not vary more than 15 per cent between two measurements close in time to the measurement $F_{s}$.

To assess force addition, parameter $A$ was defined as

$$
A=\frac{F_{s}-F_{h}}{F_{l}}
$$

Thus, $A=1$ implies full summation.

\section{Results}

\subsection{Recruitment curve measurements}

Typical recruitment curves for both electrode configurations are given in Fig. 2. Amplitudes of subsequent twitches vary at the same stimulation pulse amplitude. In 

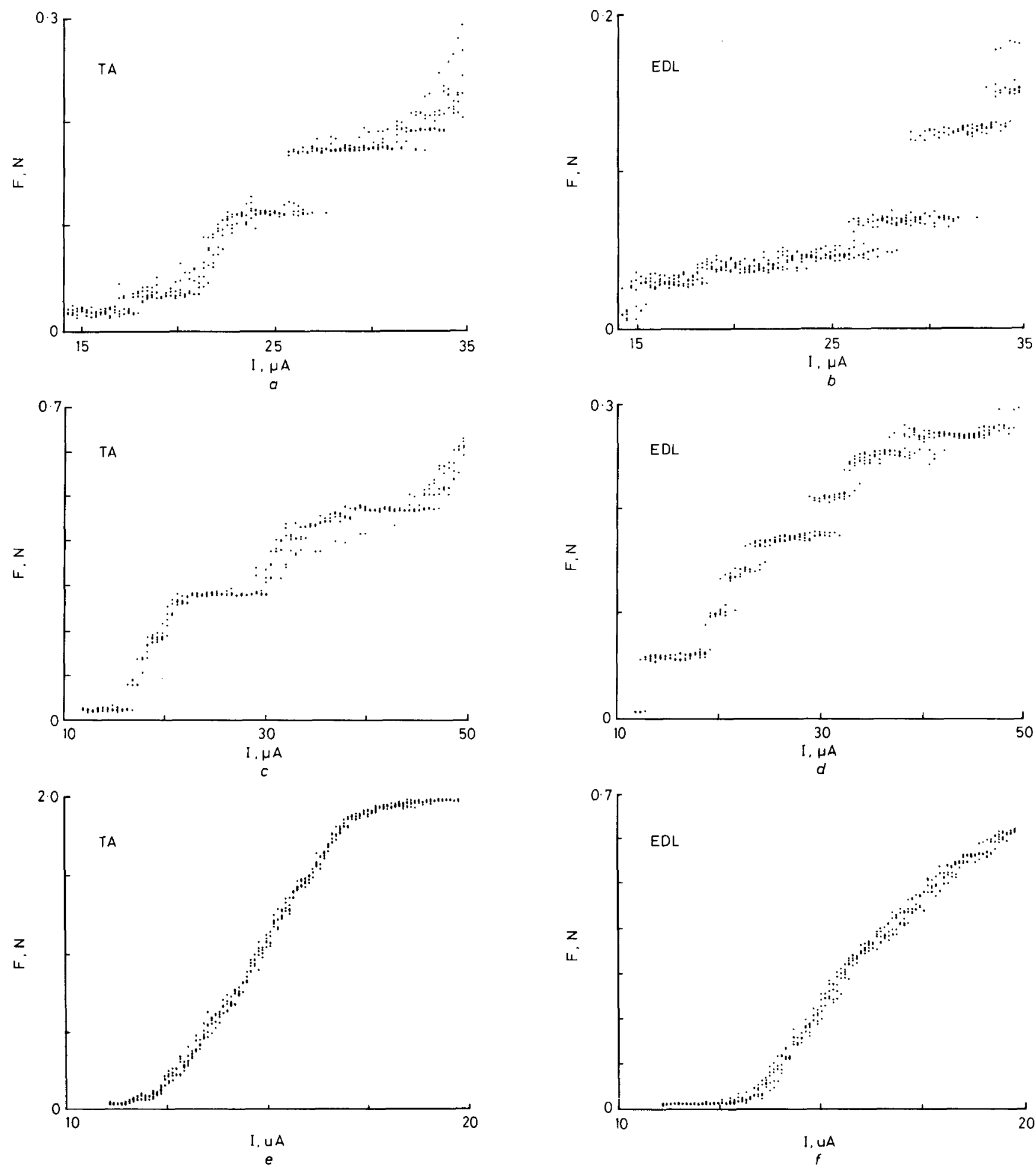

Fig. 2 Typical examples of simultaneously measured recruitment curves: (a)-(d) for intrafascicular electrodes; (e), (f) for extraneural electrodes. Figs. ( $a)$ and $(b),(c)$ and $(d),(e)$ and $(f)$ were measured simultaneously

Figs. $2 a-d$ discrete levels with varying distances in between can be observed for intrafascicular electrodes. In contrast, in almost all recordings for the extraneural electrodes no clear levels were visible (Figs. $2 e-f$ ). Table 1 specifies the number of recordings with and without clearly discernible levels for TA and EDL.

Equal probability of obtaining discrete levels under two conditions can be tested by the double dichotomy test (FreUDENTHAL, 1965), when assuming the process of obtaining clearly discernible levels or not in the recruitment curves to be a Bernouilli process. The probability of obtaining discrete levels appeared to be higher for EDL than for TA (reliability $r=95$ per cent) in the case of intrafascicular electrodes, but not in the case of extraneural electrodes. Discrete levels were found with a higher prob-
Table 1 Number of recordings with and without clearly discernible levels. Only recruitment curves were considered, for which twitch amplitudes were measured at more than 12 stimulation pulse amplitudes between $I_{t}$ and $I_{h}$

\begin{tabular}{cccc}
\hline Electrode & Muscle & $\begin{array}{c}\text { Discrete levels: } \\
\text { clearly discernible }\end{array}$ & $\begin{array}{c}\text { Discrete levels not } \\
\text { clearly discernible }\end{array}$ \\
\hline Intrafascicular & TA & 8 & 14 \\
Extraneural & EDL & 17 & 2 \\
& TA & 0 & 15 \\
& EDL & 1 & 11 \\
\hline
\end{tabular}

ability for intrafascicular than for extraneural electrodes $(r=95$ per cent $)$ in both EDL and TA muscles.

Parameter $S$ was determined for all recruitment curves measured up to at least half maximum twitch force. Histograms of $S$ are shown in Figs. $3 a-d$. $S$ was tested to be 

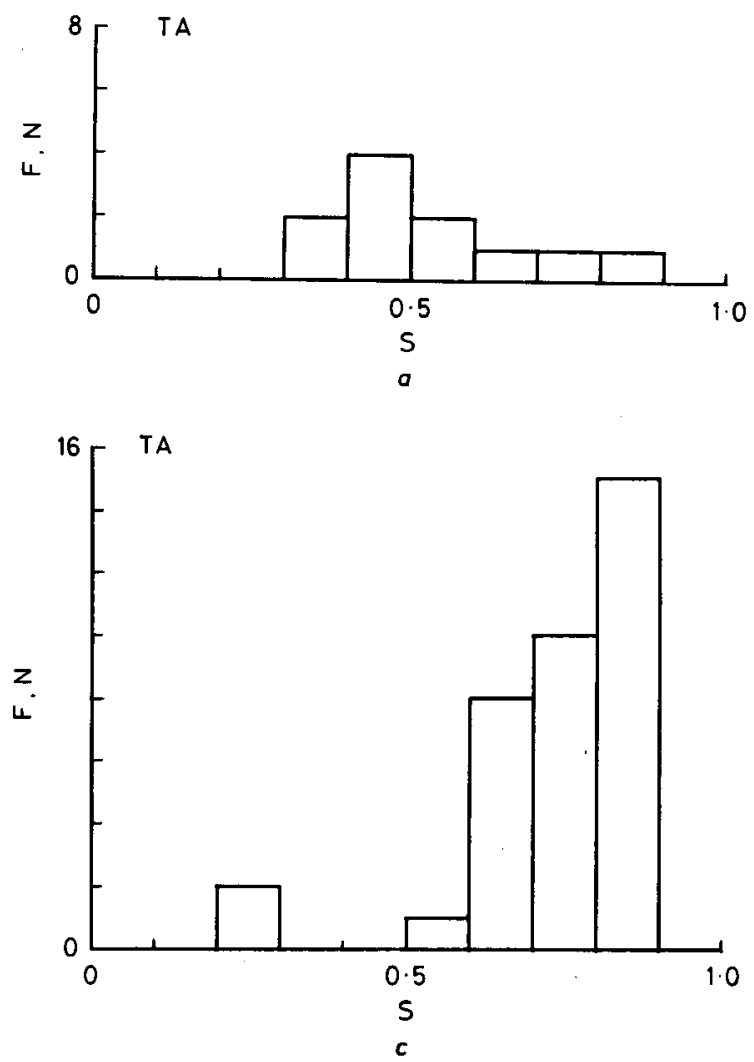

Fig. 3 Histograms of the steepness value $S$ for $T A$ and EDL: (a), (b) for intrafascicular electrodes; (c), (d) for extraneural electrodes

different under several conditions using the Wilcoxon test (HAYS, 1973). $S$ was found to be higher for extraneural than for intrafascicular electrodes for both TA and EDL muscles ( $r=95$ per cent), and appeared to be higher for EDL than for TA for both electrode configurations $(r=95$ per cent).

\subsection{Force addition measurements}

Sample responses of separate stimulation on each of both electrodes, their calculated sum, and the response of
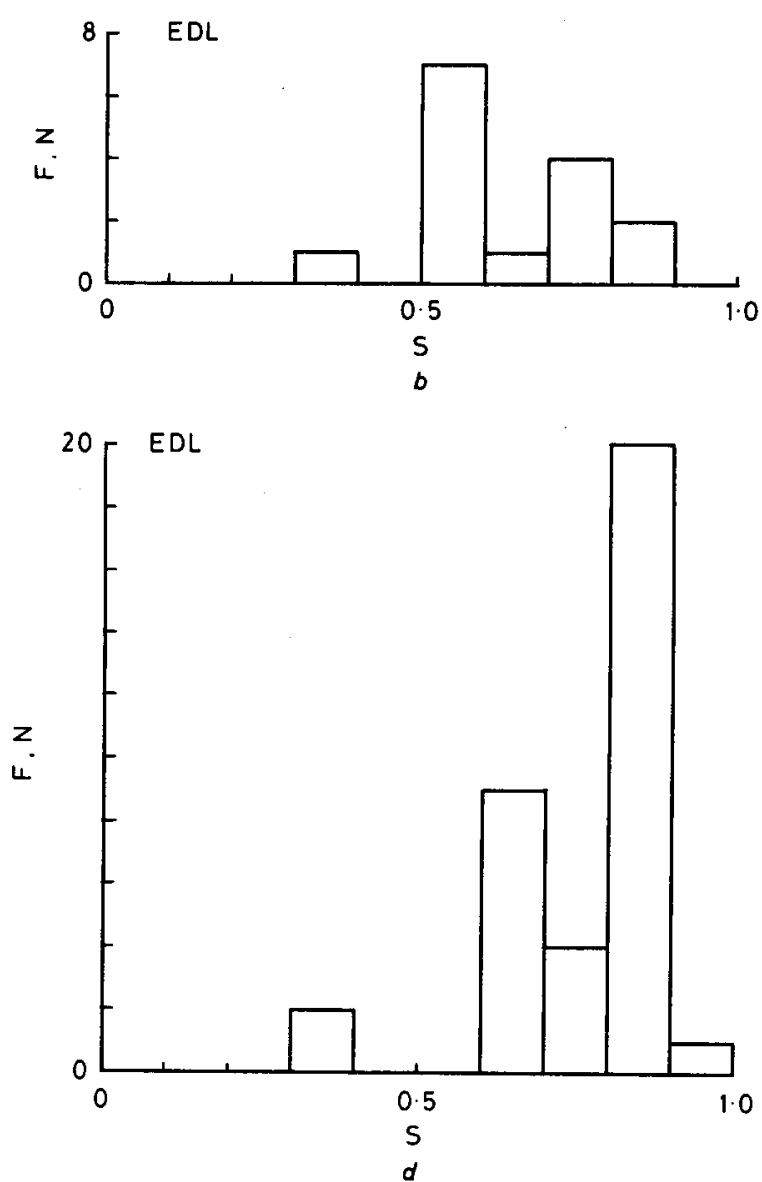

stimulating simultaneously at both electrodes are shown in Fig. 4. When no overlap of the motor unit groups recruited with both electrodes occurs, a good summation of the involved forces is to be expected (e.g. Fig. 4a). A poor force addition would indicate an extensive overlap.

The time averaged muscle force during simultaneous stimulation varied between 5 and 70 per cent of the force at supramaximal stimulation for extraneural electrodes and between 5 and 40 per cent of this force level for intrafascicular electrodes. No influence of force level on additivity parameter $A$ was apparent. Histograms of $A$ are

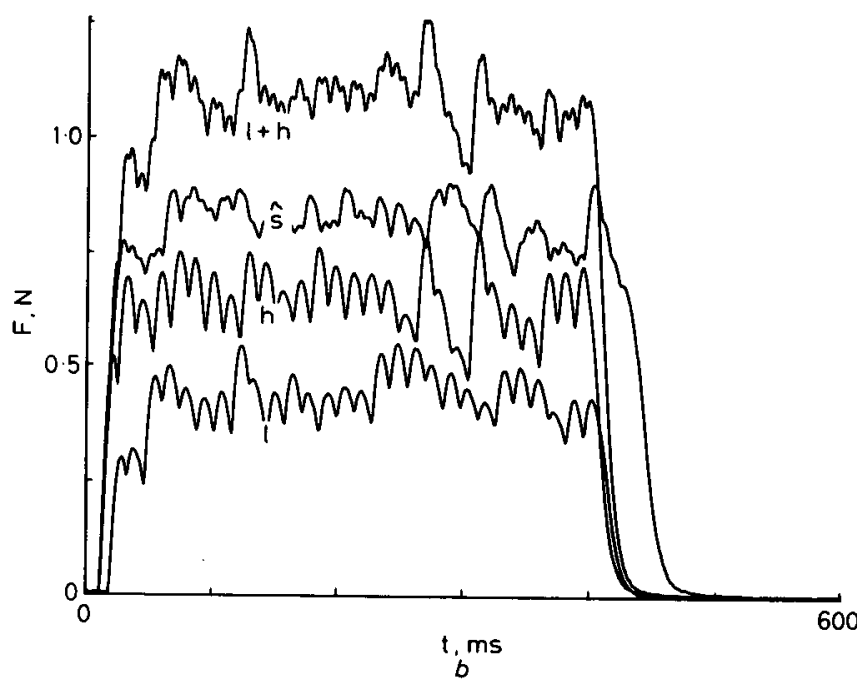

Fig. 4 Force response recordings obtained by stimulating on single electrodes or two electrodes simultaneously for the force addition experiments. During simultaneous stimulation the stimulus trains on both electrodes were $180^{\circ}$ out of phase. (a) Example for intrafascicular electrodes with good force addition; (b) typical example for extraneural electrodes

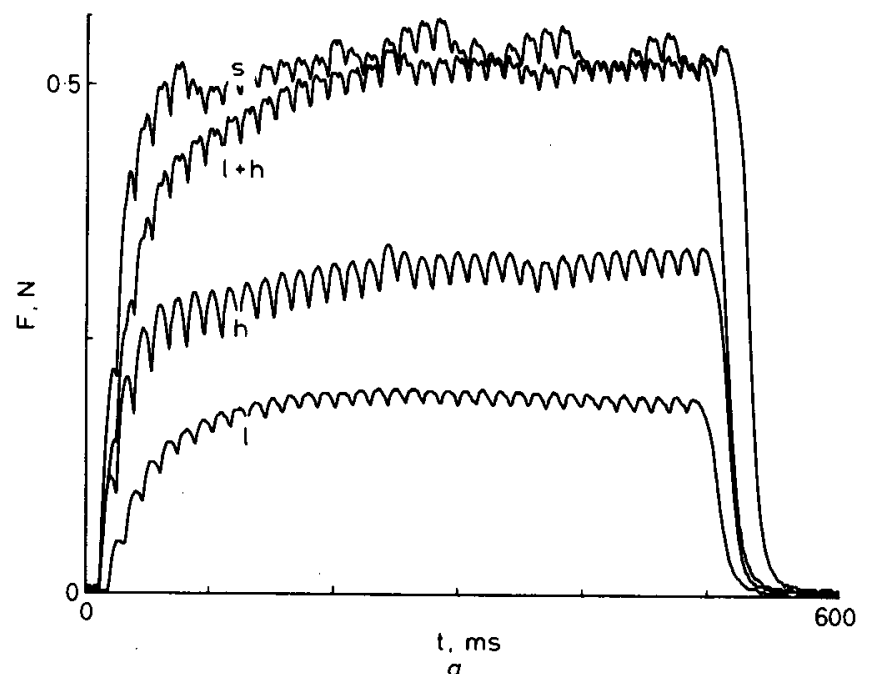

force response during stimulation on one electrode (lowest mean force)

force response during stimulation on the second electrode (highest mean force)

force response for simultaneous stimulation on both electrodes

$+h$ summation of responses $l$ and $h$ 

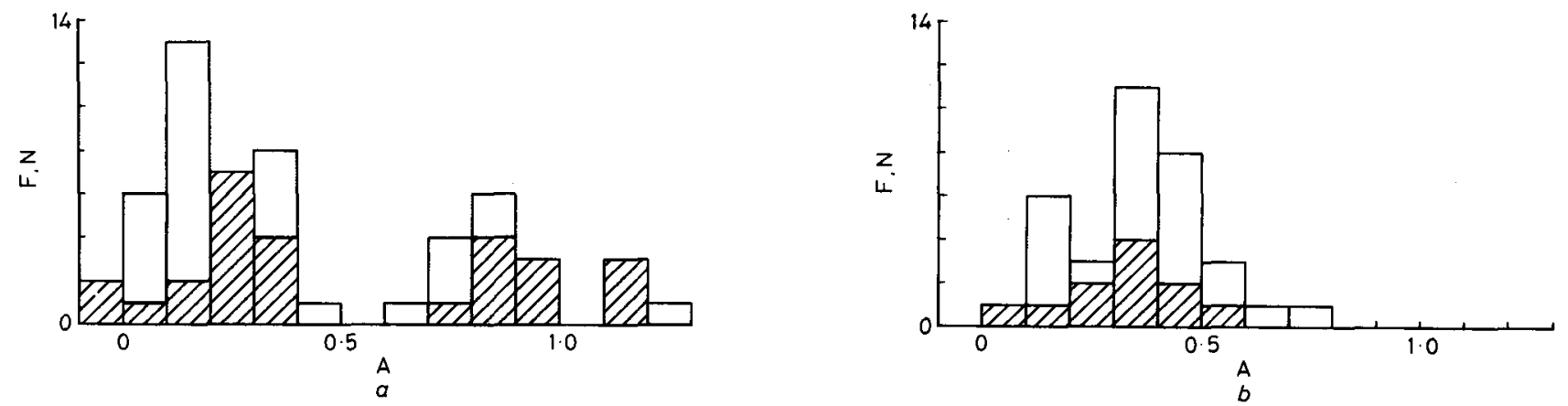

Fig. 5 Histograms of the variable A, indicating force addition: (a) for intrafascicular electrodes; (b) for extraneural electrodes adjacent electrodes non-adjacent electrodes

presented in Fig. 5. As can be seen in this figure, the mean value of $A$ does not differ much between intrafascicular and extraneural electrodes. However, there are significantly more recordings for intrafascicular electrodes with a value of $A$ greater than 0.5 than for extraneural electrodes (double dichotomy test, $r=95$ per cent). For intrafascicular electrodes $A$ was higher for non-neighbouring electrodes than for neighbouring electrodes (Wilcoxon test, $r=95$ per cent). However, this did not hold for extraneural electrodes. Variable $A$ differed significantly between some but not all rat experiments (Wilcoxon test, $r=95$ per cent), indicating varying nerve and stimulation conditions.

\section{Discussion}

Muscle twitch amplitudes can be interpreted as the sum of the twitch amplitudes of the recruited motor units (Petrofsky, 1978). The measured recruitment curves in the present work indicate a stochastic component in the excitation thresholds of motor units, the excitation threshold being defined as the stimulus amplitude above which the motoneuron fibre corresponding to the motor unit is excited, and below which it is not excited. GORMAN and MORTIMER (1983) also reported on these threshold variations during nerve stimulation, which they ascribed to fluctuations in membrane properties, perturbations in the medium between electrode and nerve, and small electrode movements. Stochastic fluctuating membrane potentials of nodes of Ranvier were reported (VERVEEN and DERKSEN, 1968), but probably cannot account for the total fluctuation of excitation thresholds of the motor units as shown in this study. Care was taken to minimise electrode movements in our experiments, so fluctuations of motor unit excitation thresholds could be expected to be minimal for the electrode configurations used.

Stochastic fluctuation of the excitation threshold of each motor unit may lead to overlapping force levels in the recruitment curves and even to masking of discrete levels. High threshold differences between motor units will result in their subsequent recruitment and thus in more stable force recruitment levels. Mean threshold differences generally are greater when the recruitment curve is less narrow (lower value of $S$ ) in the same nerve. This leads to the expectation that narrower recruitment curves are associated with less stable motor unit recruitment, which was observed in the measured recruitment curves: recruitment curves of each muscle were steeper and showed fewer stable force levels for extraneural electrodes than for intrafascicular electrodes.

Using intrafascicular electrodes, stable force levels were found more frequently in the recruitment curves of the EDL than of the TA, despite steeper curves for EDL. Apparently, average threshold difference between two subsequently recruited motor units also increases by a smaller number of motor units in a muscle, as is the case for EDL (approximately 40 in 12-week-old rats: CLOSE, 1967) as compared with TA (approximately 75 in 10-week-old rats: IsHIHARA et al., 1987).

Intrafascicular electrodes with distinct recruitment levels (e.g. Figs. $2 a-d$ ) mostly show varying distances in between. When assuming the step from one level to the next to be caused by the additional recruitment of a motor unit, these distances can be expected to be proportionally related to the size of the corresponding motor units (PETROFSKY and PhILliPS, 1979). No clear order of recruitment based on motor unit size seems to occur for the intrafascicular electrode. This corresponds to results of model studies by VELTINK et al. $(1988 a ; b)$, which predicted deviations from inverse recruitment when the electrode was close to the nerve fibres. According to these model studies, the varying positions of the nodes of Ranvier are one of the reasons for these deviations. Petrofsky (1978) and FANG and MorTIMER (1987) found inverse recruitment for extraneural electrodes, which corresponds to our model results for extraneural electrodes at larger distances from the nerve fibres (VelTINK et al., 1988a; $b$ ). These measurements and model results indicate that recruitment order is influenced by electrode position, in addition to stimulus parameters (Gorman and MorTIMER, 1983) and blocking (PETROFSKY, 1978; SOlOMONOW et al., 1983; FANG and MORTIMER, 1987). Our model results also predicted recruitment order to be influenced by nerve geometry and electrical conductivities of the media in and around the nerve (VELTINK et al., $1988 a ; b)$.

The overlap of recruited motoneuron groups is influenced by the spread of the recruitment area of each electrode. This could explain the better force addition for non-neighbouring intrafascicular electrodes compared with neighbouring electrodes. This, however, was not found for extraneural electrodes, which indicates a larger spread of recruitment areas over the fascicle for each stimulation electrode.

In our force addition experiments on rat common peroneal nerve forces did not add very well. As illustrated in Fig. 1, this nerve consists of one main fascicle, with possibly an additional small one, surrounded by just a thin connective tissue sheath. Thus force addition was tested almost at the fascicle level. Human nerves often have much higher numbers of fascicles and much more connective tissue in between the fascicles. In this case, better force addition with electrodes outside and inside the nerve is to be expected (HoLle et al., 1974) and advantage can be made of the changing nerve cross-sectional geometry along the nerve (SuNDERLAND, 1968) by placing electrodes on different places along the nerve.

Histological examinations could give additional information about the relationship between the exact electrode position and the stimulation results. Furthermore, it can 
give information about the nerve damage caused by chronic application of stimulation electrodes. Especially the chronic use of intrafascicular electrodes needs further investigation. The results of BOWMAN and ERICKSON (1985) on chronic intrafascicular implantation of coiled wire electrodes are promising: only little nerve damage and some connective tissue growth around the electrodes were observed.

Acknowledgments - We would like to thank Mr A. J. Verloop for making the electrode configurations and $\mathrm{Mr} \mathbf{J}$. H. M. Put for his assistance in the animal experiments.

\section{References}

Bowman, B. R. and Erickson, R. C. (1985) Acute and chronic implantation of coiled wire intraneural electrodes during cyclical electrical stimulation. Ann. Biomed. Eng., 13, 75-93.

Close, R. (1967) Properties of motor units in fast and slow skeletal muscles of the rat. J. Physiol., 193, 15-55.

FANG, Z.-P. and MoRTIMER, J. T. (1987) A method for attaining natural recruitment order in artificially activated muscles. Proc. 9th Ann. Conf. IEEE Eng. in Med. \& Biol. Soc., Boston, 13th-16th Nov. 1987, 657-658.

Freudenthal, H. (1965) Probability and statistics. Elsevier.

Gorman, P. H. and Mortimer, J. T. (1983) The effect of stimulus parameters on the recruitment characteristics of direct nerve stimulation. IEEE Trans., BME-30, 407-414.

GreENE, E. C. (1968) Anatomy of the rat. Hafner.

HAYS, W. L. (1973) Statistics for the social sciences, 2nd ed. Holt, Rinehart \& Winston Inc.

Holle, J., Moritz, E., Thoma, H. and Lischka, A. (1974) Die Karusselstimulation, eine neue Methode zur elektrophrenische Langzeitbeatmung. Weiner Klinische Wochenschrift, 86, 23-27.

Ishihara, A., NaItoh, H. and Katsuta, S. (1987) Effects of ageing on the total number of muscle fibers and motoneurons of the tibialis anterior and soleus muscles in the rat. Brain Res., 435, 355-358.

MCNeal, D. R. and Bowman, B. R. (1985) Selective activation of muscles using peripheral nerve electrodes. Med. \& Biol. Eng. \& Comput., 23, 249-253.

Petrofsky, J. S. (1978) Control of recruitment and firing frequencies of motor units in electrically stimulated muscles in the cat. Ibid., 16, 302-308.

Petrofsky, J. S. (1979) Sequential motor unit stimulation through peripheral motor nerves in the cat. Ibid., 17, 87-93.

Petrofsky, J. S. and Phillips, C. A. (1979) Determination of the contractile characteristics of the motor units in skeletal muscle through twitch characteristics. Ibid., 17, 525-533.

Petrofsky, J. S. and Phillips, C. A. (1981) Impact of recruitment order on electrode design for neural prosthetics of skeletal muscle. Am. J. Phys. Med., 60, 243-253.

Solomonow, M., Eldred, E., Lyman, J. and Foster, J. (1983) Control of muscle contractile force through indirect highfrequency stimulation. Ibid., 62, 71-82.

Sunderland, S. (1968) Nerves and nerve injuries. E \& S Livingstone.

Talonen, P., Baer, G., Huhti, M. and Häkkinen. V. (1985) Control of muscle force by sequential motor unit stimulation of peripheral nerves. Med. \& Biol. Eng. \& Comput., 23, Suppl., Part 1, 396-397.

ThOMA, H., Holle, J., Moritz, E. and Stöhr, H. (1978) Walking after paraplegia - a principle concept. 6th Int. Symp. on Ext.
Control of Human Extr., Yugoslav Committee for Electronics \& Automation, Dubrovnik, Sept. 1978, 71-80.

Veltink, P. H., van Alste, J. A. and BoOM, H. B. K. (1988a) Simulation of intrafascicular and extraneural nerve stimulation. IEEE Trans., BME-35, 69-75.

Veltink, P. H., van Alste, J. A. and Boom, H. B. K. (1988b) Influences of stimulation conditions on recruitment of myelinated nerve fibres: a model study. Ibid., BME-35, (in press).

Verveen, A. A. and Derksen, H. E. (1968) Fluctuation phenomena in nerve membrane. Proc. IEEE, 6, 906-916.

Wallinga-de Jonge, W., BoOm, H. B. K., Boon, K. L., Griep, P. A. M. and LAmmeree, G. C. (1980) Force development of fast and slow skeletal muscle at different muscle lengths. Am. J. Physiol., 239, C98-C104.

WILKIE, D. R. (1950) The relation between force and velocity in human muscle. J. Physiol., 110, 249-280.

\section{Authors' biographies}

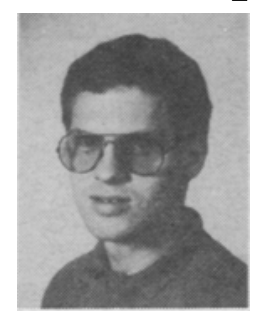

Peter H. Veltink studied Electrical Engineering at the University of Twente, Enschede, The Netherlands, where he received the MS degree in 1984 and the Ph.D. degree in 1988 (dissertation: recruitment of myelinated nerve fibres during artificial nerve stimulation). Currently, he is a member of the Biomedical Engineering Division in the Department of Electrical Engineering, University of Twente, and is engaged in research of neuromuscular stimulation for the rehabilitation of spinal-cord injured patients, which is carried out in co-operation with the Roessingh rehabilitation centre in Enschede. His research interests are nerve stimulation and control systems for functional neuromuscular stimulation.

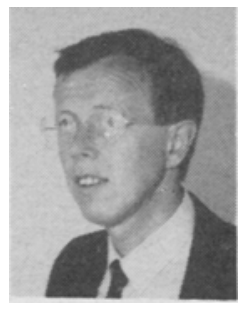

Jan A. van Alsté joined the Biomedical Engineering Division of the University of Twente after polytechnics and three years industrial electronic development. He received the MS degree in Electrical Engineering in 1978 and the Ph.D. degree in 1984, both at the University of Twente. His research interests include exercise ECG analysis, heart-assist devices and functional neuromuscular stimulation. He takes part in the rehabilitation engineering cooperation between the University of Twente and the Roessingh rehabilitation centre at Enschede, The Netherlands. Currently, he is director of the Coordination Centre for Biomedical Engineering at the University of Twente.

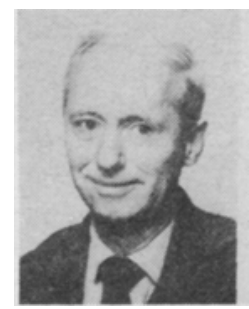

Herman B. K. Boom was trained as a medical physicist at the University of Utrecht, The Netherlands, where he received the Ph.D. degree in 1971. He joined the Departments of Medical Physics and Medical Physiology, where he was engaged in research in the field of cardiac mechanics and taught Physiology and Biophysics. Since 1976 he has occupied the Chair of Medical Electronics in the Department of Electrical Engineering, University of Twente, The Netherlands. His research interests are cardiovascular system dynamics, bioelectricity and rehabilitation technology. 